DOI: $10.24850 /$ j-tyca-2018-05-09

Artículos

\title{
Valor económico del agua de la industria manufacturera ubicada en la Subregión Hidrológica Río San Juan, México
}

Daniel A. Revollo-Fernández ${ }^{1}$

Lilia Rodríguez-Tapia ${ }^{2}$

Jorge A. Morales-Novelo ${ }^{3}$

${ }^{1}$ Conacyt-UAM, Área de Crecimiento y Medio Ambiente/Departamento de Economía, Universidad Autónoma Metropolitana, Unidad Azcapotzalco, Ciudad de México, México, darevollof@conacyt.mx,

${ }^{2}$ Departamento de Economía, Universidad Autónoma Metropolitana, Unidad Azcapotzalco, Ciudad de México, México, Irt@correo.azc.uam.mx ${ }^{3}$ Departamento de Economía, Universidad Autónoma Metropolitana, Unidad Azcapotzalco, Ciudad de México, México, jamn8647@gmail.com

Autor para correspondencia: Daniel A. Revollo-Fernández, darevollof@conacyt.mx

\section{Resumen}

El agua es uno de los insumos más importantes en el proceso de producción, sobre todo para el sector manufacturero. Pero al mismo tiempo, dicho recurso puede presentar problemas de escasez debido al desconocimiento sobre su valor. En la mayoría de países emergentes, el valor monetario que tiene dicho recurso en el proceso productivo no necesariamente refleja su escasez, así como tampoco los costos de captación y tratamiento, por lo que no se paga el costo real del agua. Este trabajo tiene como objetivo estimar el valor económico del agua en la industria manufacturera de la Subregión Hidrológica Río San Juan (SHRSJ), cuenca hídrica con problemas de escasez y de alta importancia socioeconómica, recurriendo al concepto de elasticidad de la producción con respecto al insumo agua $\left(\varepsilon_{W}\right)$ y al valor del producto marginal $\left(\rho_{w}\right)$. Para ello se revisó información de casi 18 mil unidades económicas 
encuestadas en 2013 y se consideraron dos tipos de funciones de producción: Cobb-Douglas y Trans-Logarítmicas. Se estimó que la $\varepsilon_{w}$ y el $\rho_{w}$ son iguales a 0.046 y USD $13.0 / \mathrm{m}^{3}$, respectivamente. El $\rho_{w}$ osciló entre USD $0.9 / \mathrm{m}^{3}$ para el sector de equipo de computación a USD $15.4 / \mathrm{m}^{3}$ para el sector de productos químicos, lo que indica que el $\rho_{w}$ varía según los sectores. Los resultados tienen importantes implicaciones para diversas áreas de la gestión del agua industrial, especialmente en aquellas cuencas o regiones hídricas donde dicho recurso presenta problemas de escasez $y$, al mismo tiempo, son regiones de alta importancia socioeconómica.

Palabras clave: valor económico, agua, industria manufacturera, escasez.

Recibido: 19/10/2017

Aceptado: $11 / 04 / 2018$

\section{Introducción}

Aunque la oferta de agua, en términos absolutos, se considera abundante, México enfrenta un serio problema de escasez relativa (Conagua, 2012a). Este problema se manifiesta en la incapacidad de abastecer la demanda de agua en algunas cuencas del país, sobre todo en aquellas donde, con el paso de los años, la población crece en mayor medida.

La Subregión Hidrológica Río San Juan (SHRSJ) ocupa menos de 3.3\% del territorio nacional y sin embargo en ella habita un $6.2 \%$ de la población total del país, generando cerca de $9.3 \%$ del Producto Interno Bruto (PIB) nacional. En el ámbito internacional, el grado de presión sobre el recurso hídrico se puede medir a través del Indice Relativo al Estrés Hídrico, el cual se define como la relación entre el uso del agua y la disponibilidad natural media del agua, donde los valores superiores a $40 \%$ indican la presencia de estrés hídrico severo. En el caso de SHRSJ, ésta presenta un valor igual a $77.1 \%$, que la ubica dentro de las 
regiones con más alto nivel de presión sobre sus recursos hídricos en México (Conagua, 2016). En ese sentido, en un contexto de estrés hídrico severo, es imprescindible que se adopten políticas que busquen que los diversos usos del agua que se presentan en las regiones y subregiones sean eficientes. En la actualidad y con base en su importancia, los principales sectores que consumen agua proveniente de la SHRSJ son el público, urbano-doméstico, agrícola e industrial (Aguilar-Benitez, 2017).

La industria manufacturera tiene relevancia clave en la dinámica de la actividad económica de la SHRSJ, pues explica dos quintas partes de su PIB, generando fuentes de empleo, y bienes y servicios que se destinan al consumo de la población de la región, y a la exportación fuera de la subregión hidrológica y del país (Conagua, 2012b). El agua es un recurso esencial en el proceso de producción de la industria que está ubicada en la cuenca; sólo para el año 2015 incorporó a su proceso productivo unos 99 millones de $\mathrm{m}^{3}$. Este volumen representa el $7.7 \%$ del agua que utiliza la industria en el país (Conagua, 2016). Por razones de su propio proceso productivo, la industria manufacturera extrae cerca de un $56 \%$ de agua de fuentes subterráneas (acuíferos), los que a la fecha registran una sobreexplotación y, por ende, están declarados en zona de veda, y reportan disponibilidad nula del recurso, ocasionando daños irreversibles sobre los mismos. La industria complementa sus requerimientos de agua del suministro público urbano que en su mayoría se abastece de agua del subsuelo, con los mismos impactos adversos en los mismos cuerpos de agua (Aguilar-Benitez, 2017). Esta sobreexplotación de acuíferos, tanto la generada por la industria manufacturera como de otros sectores económicos, genera de igual manera, efectos perjudiciales a la sociedad, como el agotamiento de manantiales, desaparición de lagos y humedales, eliminación de vegetación nativa y pérdida de ecosistemas, disminución del rendimiento de los pozos, incremento en los costos de extracción, asentamiento y agrietamiento del terreno, entre otros.

Sin embargo, como en la mayoría de los países o zonas emergentes o en vías de desarrollo, el valor monetario que tiene dicho recurso natural en el proceso productivo no necesariamente refleja su escasez, así como tampoco los costos de captación y tratamiento que requiere, por lo que no se paga el costo real del agua y esto no permite tomar conciencia de su ineficiente uso, malgasto o contaminación (Dupont \& Renzetti, 2001). Asimismo, por lo general, en la mayoría de ciudades del país, el precio 
del agua ha sido regulado por el gobierno del estado, por lo que la importancia del valor del agua industrial ha sido pasada por alto.

El uso del agua en la industria manufacturera adiciona valor al producto o servicio final, y este valor adicional es el valor del agua que se incorpora en su proceso, al reflejar el pago efectivo de los consumidores por el uso del recurso. La estimación del valor monetario adicional del producto final, que agrega un metro cuadrado adicional de agua a los productos elaborados (valor marginal del agua) en la Subregión Hidrológica Río San Juan (SHRSJ), es el objetivo del presente artículo; del mismo modo se identifican las industrias que suman más o menos valor al recurso.

Por otro lado, conocer el valor económico del agua (VEA) permite una correcta y eficiente gestión del recurso (Dupont \& Renzetti, 2001). En primer lugar, ofrece una herramienta para aquellos que toman decisiones con respecto a los temas de agua para la ejecución de inversiones futuras sustentadas en criterios técnicos, tomando en cuenta que las ciudades o países continúan invirtiendo en recursos hídricos, constituyendo uno de los principales componentes dentro de su presupuesto para infraestructura pública. En segundo lugar, la evaluación económica es importante, porque permite determinar si la sociedad, en este caso las empresas, están en favor o no de proyectos destinados para el abastecimiento de agua.

Por último, el VEA es útil para evaluar el diseño e implementación de política pública, no necesariamente referida a inversión en construcción sino a un manejo más eficiente del agua.

La literatura internacional sobre estimaciones del VEA para países en vías de desarrollo o emergentes con problemas de escasez es relativamente limitada: está enfocada a sectores agrícolas y domésticos. En tal contexto, este artículo pretende llenar dicho vacío, al estimar su valor en la industria manufacturera de la Subregión Hidrológica Río San Juan (SHRSJ), subregión que enfrenta un relativo estrés hídrico y un constante desarrollo socioeconómico.

La estructura de este artículo, además de la introducción, incluye seis apartados. En la siguiente sección se presenta una revisión de literatura, seguida por una sección donde se provee información sobre el lugar de estudio. Posterior a esa sección se presentan la metodología y los resultados obtenidos. Finalmente, en el sexto apartado, se desarrollan las conclusiones y recomendaciones. 


\section{Revisión de literatura}

En el proceso de producción de bienes y servicios que se destinan a la economía se requiere de una serie de combinaciones de recursos o insumos como equipos, mano de obra, gestión, capital, espacio y recursos naturales, entre otros, que contribuyen al valor total de la producción (Ku \& Yoo, 2012) (OJO AUTOR, REVISAR SI SON ÉSTOS LOS AUTORES, VER NOTA EN REFERENCIAS). En el caso del sector industrial manufacturero, el valor que éste otorga al recurso agua es clasificado como valor de uso directo y valorado por medio del precio de mercado, al cual la industria tiene acceso (Marcouiller \& Coggins, 1999); sin embargo, no necesariamente el precio de los productos de mercado, en este caso el agua, refleja su verdadero valor. Dentro de la teoría económica existen tres enfoques que tratan de estudiar y calcular este valor de la producción (Ku \& Yoo, 2012). Primero, los que determinan la demanda de agua; segundo, los que estudian la estructura de costos; y tercero, los que investigan la estructura de producción.

En el primer enfoque, los métodos de estimación de la demanda del agua consisten en regresiones estadísticas directas, tratando de estudiar la relación de la cantidad consumida del recurso como variable dependiente, y como independientes el precio del agua, la producción, el trabajo y otras variables socioambientales (Rees, 1969; Oh, 1973; DeRooy, 1974; William, 1986). Sin embargo, la teoría establece que este enfoque genera un sesgo por la cantidad de agua consumida que está en ambos lados de la regresión, generando resultados poco consistentes y relativamente discutibles (Hussaina, Thrikawalaa, \& Barkera, 2002).

Para tratar de solucionar tal inconveniente, investigaciones más recientes emplean la misma metodología, pero asumiendo diferentes formas funcionales de los modelos (Malla \& Gopalakrishnan, 1999; Onjala, 2001; Hussaina et al., 2002) u otros métodos de estimación como sistema en dos o tres etapas (Bruneau, Renzetti, \& Villeneuve, 2010; Féres, Reynaud, \& Thomas, 2012). 
El segundo enfoque, a través de la estructura de costos, relaciona los costos totales de la actividad como variable dependiente y los precios de los distintos factores productivos, como el agua y la mano de obra, entre otros, y la producción como regresores (Grebenstein \& Field, 1979; Féres, \& Reynaud 2003; Reynaud, 2003).

Por último, el tercer enfoque recurre a una función de producción, que relaciona el valor de la producción como variable dependiente y el valor monetario o cantidades físicas de los distintos factores productivos (Wang \& Lall, 2002; Ku \& Yoo, 2012).

En el caso de este último enfoque, la metodología permite recuperar la productividad marginal por cada factor productivo, que corresponde al precio implícito de mercado de este recurso, lo cual es el mayor aporte -a diferencia de los dos primeros enfoques-, recomendable para aquellos casos de estudio donde los precios de los insumos no necesariamente reflejen su verdadero valor; esto, por ejemplo, a consecuencia del establecimiento de políticas públicas o de mercado que intentan dar beneficios a las unidades económicas, como es el caso del establecimiento de subsidios.

Dentro de la literatura económica, los dos primeros enfoques son los más utilizados. Sin embargo, requieren del establecimiento de un mercado de agua, lo cual se traduce en fijar una estructura tarifaria adecuada e implementada de manera correcta, y con ella datos de costos y precios marginales de los insumos (De-Gispert, 2004). No obstante, precios medios son usados como proxys de los precios marginales.

Empero, como indican Dupont y Renzetti (2001), ello se traduce en un sesgo deliberado, pues en competencia perfecta, las firmas alcanzan su nivel óptimo en los precios marginales. Por otro lado, el mercado del agua al que se enfrentan las industrias depende de la fuente de origen del agua que éstas tengan. Así, por ejemplo, cuando se trata de autoabastecimiento, se tiene poco o ningún costo externo; al contrario, cuando es suministrada desde la red pública, las empresas se enfrentan a una estructura de precios en bloques crecientes (Renzetti \& Dupont, 2002).

Ahora bien, si una simplificación del mercado no es posible -es decir, no se pueden utilizar precios medios y se desconoce la estructura tarifaria- o se evidencia que si bien existe una estructura tarifaria no está correctamente implementada, el enfoque de productividad marginal puede ser usado para evaluar el valor marginal del agua industrial, pues 
no necesita precios de los insumos. En ese sentido, la presente investigación recurre al tercer enfoque.

Por otro lado, el número de investigaciones sobre el VEA en uso industrial a nivel mundial es menor que el de los usos agrícolas y domésticos; mientras que en el caso nacional es casi nulo. Esto, debido a que los precios de los insumos, como el agua que se utiliza en la industria, por lo general presentan subsidios y las políticas de precios que no son en su totalidad de mercado.

Algunos estudios donde utilizan el tercer enfoque, como el de Wang y Lall (2002), estiman el valor marginal del agua para una muestra de unas 2000 empresas del sector industrial en China, que va desde USD $0.02 / \mathrm{m}^{3}$ para el sector de electricidad, hasta USD $11.5 / \mathrm{m}^{3}$ para el sector de equipo de transporte, con un valor promedio nacional de USD $1.1 / \mathrm{m}^{3}$ para toda la industria.

Por otro lado, Aylward, Seely, Hartwell y Dengel (2010), a través de una revisión de investigaciones efectuadas en diversos países de Asia y Norteamérica, excepto México, estiman que para el sector industrial, el valor de la productividad marginal del agua presenta un margen de USD $0.01 / \mathrm{m}^{3}$ a USD $6.94 / \mathrm{m}^{3}$.

De igual forma, Ku y Yoo (2012) (OJO AUTOR, REVISAR SI SON ÉSTOS LOS AUTORES, VER NOTA EN REFERENCIAS) estiman el valor de la productividad marginal del agua para la industria manufacturera en Corea con un valor promedio para toda la industria igual a USD $3.7 / \mathrm{m}^{3}$; el menor valor es el del sector de instrumentos de precisión y el mayor el sector de equipo de transporte, con USD 1.4/ $\mathrm{m}^{3}$ y USD $44.3 / \mathrm{m}^{3}$, respectivamente.

Finalmente, Vargas (2015) presume un valor marginal igual a USD $30.9 / \mathrm{m}^{3}$ para la industria manufacturera de Chile, con un rango entre USD $11.8 / \mathrm{m}^{3}$ y USD $75.3 / \mathrm{m}^{3}$ para el sector textil, y de metales comunes, respectivamente.

\section{Subregión Hidrológica Río San Juan (SHRSJ)}




\section{Ubicación Subregión Hidrológica Río San Juan (SHRSJ)}

Para fines administrativos y de preservación de las aguas nacionales, a partir de 1997, México se dividió en 13 Regiones HidrológicasAdministrativas (RHA), formadas por agrupaciones de cuencas, consideradas las unidades básicas de gestión de los recursos hídricos.

Las RHA presentan límites que respetan los municipios para facilitar la integración de la información socioeconómica.

La Subregión Hidrológica Río San Juan (SHRSJ) forma parte de la Región Hidrológica Bravo-Conchos y de la Región Hidrológica-Administrativa Río Bravo (RHA número 6) (Figura 1).

La SHRSJ tiene una superficie de $30847.26 \mathrm{~km}^{2}$, abarcando municipios de los estados de Coahuila (cuatro municipios); Nuevo León (37 municipios), y Tamaulipas (ocho municipios). Los principales municipios de esta subregión son Saltillo, que se ubica en Coahuila; Monterrey, Guadalupe y Apodaca, en Nuevo León; Reynosa y Matamoros, en Tamaulipas. Presenta gran actividad económica y la población suma más de medio millón de habitantes.

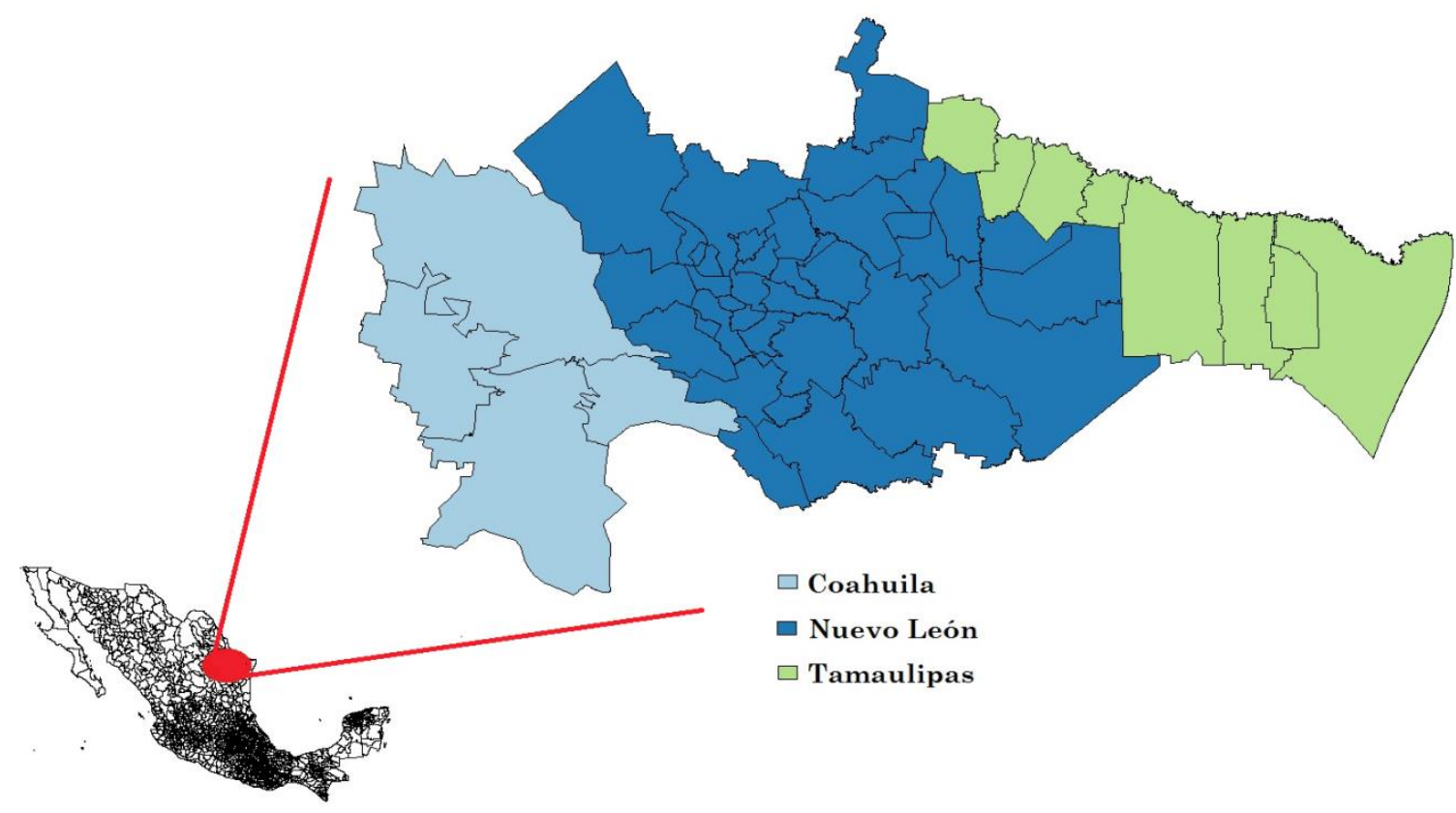


Figura. 1. Delimitación hidrológica de la Subregión Hidrológica Río San Juan (SHRSJ). Fuente: elaboración propia, 2017.

La SHRSJ es una de las regiones más densamente poblada en México, con unos 7.2 millones de habitantes $(12.8 \%$ en Coahuila; $19.9 \%$ en Tamaulipas; $67.3 \%$ en Nuevo León), con alrededor de 236 habitantes por $\mathrm{km}^{2}$, siendo una de las más densas a nivel nacional. Ocupa menos de $3.3 \%$ del territorio nacional, pero donde habita cerca de $6.2 \%$ de la población total del país, generando un $9.3 \%$ del PIB nacional.

\section{La industria manufacturera en la Subregión Hidrológica Río San Juan (SHRSJ)}

El sector manufacturo en la SHRSJ alcanza un número de 17983 unidades económicas (UE), que representan el $3.7 \%$ nacional del sector manufacturero, generando $17.1 \%$ del valor del producto de dicho sector en el país, y emplea a unos 700000 trabajadores, que representan casi el $25.1 \%$ de la población ocupada del sector industrial en dicha subregión y un $13.6 \%$ de la ocupación nacional del sector industrial. En ese sentido se observa la alta productividad de la zona con respecto a la situación nacional.

La importancia socioeconómica y el dinamismo del sector manufacturero en la SHRSJ sugiere investigar el impacto que tiene su demanda de agua como insumo productivo, dada su ubicación en una región caracterizada por escasez absoluta del recurso.

El sector manufacturero incorpora agua en su proceso, en la modalidad de uso consuntivo, lo cual indica que el agua extraída del cuerpo de agua no retorna al mismo. El volumen de agua anual que demanda todo el sector manufacturero es de $99 \mathrm{hm}^{3}$ de agua de primer uso, la mayoría extraída de pozos localizados en la misma cuenca (56\%) y en segundo lugar de fuentes superficiales (44\%).

En la Tabla 1 se describen para cada una de las industrias presentes en la subregión su respectiva participación dentro del agua utilizada por el 
sector industrial y dentro de la producción manufacturera anual, información que muestra la intensidad de cada industria en los recursos hídricos y su importancia en la actividad económica de la región. Las industrias que ejercen mayor intensidad sobre los recursos hídricos en la SHRSJ son ocho: 1) productos eléctricos; 2) productos metálicos; 3) equipo de computación; 4) química; 5) equipo de transporte; 6) plástico y hule; 7) alimentación; 8) bebidas y tabaco. Estas industrias se agrupan bajo la etiqueta de industrias de alta intensidad, porque juntas explican un poco más de tres cuartas partes de toda el agua requerida cada año por el sector manufacturero y exactamente tres cuartas partes del valor de la producción. Dentro de las industrias de alta intensidad, destacan las de aparatos eléctricos y productos metálicos; ambas participan con el 33\% de uso del agua. En el lado opuesto se ubica la industria de bebidas y tabaco, con un consumo de $4.7 \%$ del total de agua de la industria manufacturera.

Tabla 1. Intensidad de la industria manufacturera en el uso de recursos hídricos en la Subregión Hidrológica Río San Juan (SHRSJ). Fuente: elaboración propia, 2017.

\begin{tabular}{|c|c|c|c|}
\hline \multicolumn{2}{|c|}{ Clasificación de la industria } & $\begin{array}{c}\text { Participación en el } \\
\text { uso del agua }\end{array}$ & $\begin{array}{c}\text { Participación en el } \\
\text { valor de la } \\
\text { producción }\end{array}$ \\
\hline Subsector & Subsector & \% & \% \\
\hline $\mathbf{3 3 5}$ & Aparatos eléctricos & 20.83 & 7.95 \\
\hline $\mathbf{3 3 2}$ & Productos metálicos & 12.76 & 8.13 \\
\hline $\mathbf{3 3 4}$ & Equipo de computación & 11.63 & 1.68 \\
\hline $\mathbf{3 2 5}$ & Química & 10.62 & 8.40 \\
\hline $\mathbf{3 3 6}$ & Equipo de transporte & 10.04 & 30.05 \\
\hline $\mathbf{3 2 6}$ & Plástico y hule & 7.03 & 5.32 \\
\hline $\mathbf{3 1 1}$ & Alimentario & 5.75 & 7.90 \\
\hline $\mathbf{3 1 2}$ & Bebidas y tabaco & 4.70 & 4.62 \\
\hline Grupo & Alta intensidad & 83.36 & 74.05 \\
\hline $\mathbf{3 2 7}$ & Minerales no metálicos & 4.17 & 3.68 \\
\hline $\mathbf{3 2 2}$ & Papel & 2.19 & 2.74 \\
\hline $\mathbf{3 3 1}$ & Metálicas básicas & 2.80 & 11.94 \\
\hline $\mathbf{3 3 3}$ & Maquinaria y equipo & 2.55 & 5.45 \\
\hline $\mathbf{3 3 9}$ & Otras industrias & 2.06 & 0.60 \\
\hline
\end{tabular}




\begin{tabular}{|c|c|c|c|}
\hline $\mathbf{3 3 7}$ & Muebles & 1.82 & 0.63 \\
\hline $\mathbf{3 2 3}$ & Impresión & 0.57 & 0.36 \\
\hline $\mathbf{3 1 5}$ & Prendas de vestir & 0.32 & 0.25 \\
\hline $\mathbf{3 2 1}$ & Madera & 0.09 & 0.19 \\
\hline $\mathbf{3 1 4}$ & Productos textiles & 0.04 & 0.06 \\
\hline $\mathbf{3 1 3}$ & Insumos textiles & 0.02 & 0.03 \\
\hline $\mathbf{3 1 6}$ & Cuero y piel & 0.01 & 0.02 \\
\hline Grupo & Intensidad normal & 16.6 & 26.0 \\
\hline & Sector & $\mathbf{1 0 0 . 0}$ & $\mathbf{1 0 0 . 0}$ \\
\hline
\end{tabular}

Las doce industrias restantes presentes en la subregión no presionan en forma importante los recursos hídricos, pues en conjunto demandan un $16.6 \%$ de toda el agua requerida cada año por el sector manufacturero y producen un cuarto del valor de la producción de dicho sector. Este resultado se presenta aun cuando incluye el sector de minerales no metálicos, cuya tecnología requiere un $4.2 \%$ del agua total de la industria.

Las industrias que presentan mayor intensidad de los recursos hídricos en la SHRSJ deben mejorar su eficiencia en el uso del agua; necesitan aplicar cambios esenciales, incluyendo mejoras tecnológicas. Esto es factible de llevar a cabo si el valor marginal del agua utilizada por industria es lo suficientemente elevada como para generar los ingresos que dichos ajustes implican, al mismo tiempo que permita estimar la factibilidad de internalizar las externalidades que están produciendo en los cuerpos de agua de la SHRSJ. Este escenario de la SHRSJ se completa con el valor marginal del agua en las industrias que se realiza en este trabajo.

\section{Metodología}

\section{Modelo}


La función de producción tipo Cobb-Douglas (CD), por lo general se emplea en el análisis empírico de los mercados de factores, y de producción de bienes y servicios. Sin embargo, esta función presenta restricciones desarrolladas y estudiadas con el paso del tiempo debido al supuesto de aditividad y homogeneidad. En ese sentido, Christensen, Jorgenson y Lau (1973) propusieron la función Trans-Log (TL) para superar estas restricciones y demostraron que por medio de ésta se proporciona una gama más amplia de sustitución de patrones de transformación, que aquellos restringidos por la elasticidad constante de la sustitución, como es el caso de la función CD (Wang \& Lall, 2002).

Como indican Baumann, Boland y Hanemann (1997), la función CD requiere que todos insumos de la función de producción sean sustitutos, y esto limita el grado de sustitución al ser un valor constante y el mismo para todos, más conocido como elasticidad de sustitución unitaria; situación contraria a lo que sucede con una función de producción TL. Por lo tanto, en el presente estudio se consideran ambos tipos de funciones de producción para poder comparar resultados.

\section{Modelo función Cobb-Douglas (CD)}

La función de producción para un bien o servicio, donde se considera como un insumo el recurso agua, puede ser representada como $Q=f$ $(K, L, W, M)$, donde $Q$ es la producción final del bien o servicio analizado; $K$, el capital empleado; $L$, el trabajo utilizado; $W$, el agua usada en la producción, y $M$ es el resto de insumos intermedios en el proceso de producción. Por lo tanto, la función de producción donde se considera el capital, trabajo utilizado, agua y materiales como insumos puede representarse como:

$\ln Q=\ln A+\alpha_{1} * \ln L+\alpha_{2} * \ln W+\alpha_{3} * \ln M+\alpha_{4} * \ln K+e(1)$ 
En ese sentido, la elasticidad de la producción puede calcularse a través de la derivada parcial con respecto a cada uno de los factores considerados. Para el caso del recurso agua, la elasticidad $\left(\varepsilon_{C D-w}\right)$ se obtiene como:

$\varepsilon_{\mathrm{CD}-\mathrm{W}}=(\partial Q / Q) /(\partial W / W)=\alpha_{2}$

Mientras que el valor marginal del agua $\left(\rho_{C D-w}\right)$ se calcula como:

$$
\rho_{\mathrm{CD}-\mathrm{W}}=(\partial Q / \partial W)=\varepsilon_{\mathrm{CD}-\mathrm{W}} *(Q / W)
$$

\section{Modelo función Trans-Logarítmica (TL)}

Después de tomar logaritmos en ambos lados a la función de producción CD y aplicar una expansión de Taylor de segundo orden, la función de TL se representa como:

$\ln Q=\ln A+\alpha_{1} * \ln L+\alpha_{2} * \ln W+\alpha_{3} * \ln M+\alpha_{4} * \ln K+\alpha_{5} * \ln L * \ln W+\alpha_{6} *$ $\ln L * \ln M+\alpha_{7} * \ln L * \ln K+\alpha_{8} * \ln W * \ln M+\alpha_{9} * \ln W * \ln K+\alpha_{10} * \ln M * \ln K+$ $\alpha_{11} *(\ln L)^{2}+\alpha_{12} *(\ln W)^{2}+\alpha_{13} *(\ln M) 2+\alpha_{14} *(\ln K)^{2}+e(4)$

Donde la elasticidad de la producción con respecto al insumo agua ( $\varepsilon_{T L-}$ w) se obtiene como:

$\varepsilon_{\mathrm{TL}-\mathrm{W}}=(\partial Q / Q) /(\partial W / W)=\alpha_{2}+\alpha_{5} * \ln L+\alpha_{8} * \ln M+\alpha_{9} * \ln K+2 * \alpha_{12} * \ln W$ (5)

Y el valor marginal del agua $\left(\rho_{T L-W}\right)$ como: 
$\rho_{\mathrm{TL}-\mathrm{W}}=(\partial Q / Q)=\varepsilon_{\mathrm{TL}-\mathrm{W}} *(Q / W)=\left(\alpha_{2}+\alpha_{5} * \ln L+\alpha_{8} * \ln M+\alpha_{9} * \ln K+2 *\right.$ $\left.\alpha_{12} * \ln W\right) *(Q / W)(6)$

\section{Datos}

Los datos se obtuvieron del Censo Económico Industrial del Instituto Nacional de Estadística y Geografía (INEGI), a través del Sistema Automatizado de Información Censal (SAIC). El Censo Económico se aplicó a las unidades económicas (UE) que se dedican a realizar diversas actividades industriales, y se recolecta información como sueldos y salarios, el valor y volumen de la producción, así como la maquinaria y el equipo, entre otros.

La información fue procesada para las UE del sector manufacturero (códigos 31, 32 y 33), ubicadas en los municipios de la Subregión Hidrológica Río San Juan (SHRSJ) (Coahuila de Zaragoza, Nuevo León y Tamaulipas). Las UE manufactureras de cada municipio se agregaron por subsector y se obtuvieron unas 1010 observaciones por variable para el año 2014. La agregación se debe a que por la Ley de Confidencialidad que maneja el Instituto Nacional de Estadística y Geografía (INEGI), la información no puede mostrarse en forma individualizada, a fin de evitar que se utilice con fines fiscales, judiciales o administrativos y existen industrias con poca información a nivel municipal. En total, se captó información de alrededor de 17983 UE repartidas en sectores económicos, que se agregan en 49 municipios que integran la SHRSJ.

Las variables utilizadas para estimar la función de producción son: a) valor de productos elaborados $(Q)$, que cuantifica el valor monetario de todos los bienes y servicios producidos o comercializados por la UE como resultado del ejercicio de sus actividades; b) personal ocupado total (L), que comprende a todas las personas que trabajaron durante el periodo de referencia sujetas a su dirección y control; c) materias primas y materiales que se integran a la producción (M), es el valor monetario a costo de adquisición en el mercado nacional o extranjero de las materias primas y auxiliares consumidas en los procesos de producción; d) agua utilizada en la producción $\left(\mathrm{m}^{3}\right)(W)$, se partió de la información del 
gasto monetario en agua reportada en el censo y se dividió por su correspondiente precio por $\mathrm{m}^{3}$ de consumo de agua; e) acervo total de activos fijos $(K$,$) , se tomó el valor actualizado de todos aquellos bienes$ propiedad de la UE que tienen la capacidad de producir o proporcionar las condiciones necesarias para la generación de bienes y servicios.

Para la estimación de la función se construyó una base de información de las respectivas variables $(Q, L, M, W$ y $K$ ) para los diferentes sectores (códigos 31, 32 y 33) a nivel municipal. Dentro la industria manufacturera se contemplan los siguientes subsectores (Tabla 2): a) alimentos (código 311); b) bebidas y tabaco (código 312); c) fabricación de insumos textiles y acabado de textiles (código 313); d) fabricación de productos textiles, excepto prendas de vestir (código 314); e) fabricación de prendas de vestir (código 315); f) curtido y acabado de cuero y piel, y fabricación de productos de cuero piel y materiales sucedáneos (código 316); g) industria de la madera (código 321); h) industria del papel (código 322); i) impresión de industrias conexas (código 323 ; j) fabricación de productos derivados del petróleo y del carbón (código 324); k) industria química (código 325); I) industria del plástico y del hule (código 326); m) fabricación de productos a base de minerales no metálicos (código 327); n) industrias metálicas básicas (código 331); o) fabricación de productos metálicos (código 332); p) fabricación de maquinaria y equipo (código 333); q) fabricación de equipo de computación comunicación medición, y de otros equipos componentes y accesorios electrónicos (código 334); r) fabricación de accesorios para aparatos eléctricos y equipo de generación de energía eléctrica (código 335); s) fabricación de equipo de transporte (código 336 ); t) fabricación de muebles colchoneros y persianas (código 337), y u) otras industrias (código 339).

Tabla 2. Clasificación industrial por subsectores de la industria manufacturera. Fuente: elaboración propia, 2017.

\begin{tabular}{|c|c|l|}
\hline Subsector & Código & \multicolumn{1}{|c|}{ Descripción } \\
\hline Alimentos & 311 & $\begin{array}{l}\text { UE dedicadas a la elaboración, conservación y } \\
\text { envasado de productos alimentarios para consumo } \\
\text { humano y para alimentos }\end{array}$ \\
\hline Bebidas y tabaco & 312 & $\begin{array}{l}\text { UE dedicadas a la elaboración de bebidas alcohólicas } \\
\text { y no alcohólicas, al beneficio del tabaco y a la } \\
\text { elaboración de productos de tabaco; incluye la } \\
\text { purificación de agua, en donde se llena directamente } \\
\text { el garrafón de los clientes }\end{array}$ \\
\hline
\end{tabular}




\begin{tabular}{|c|c|c|}
\hline $\begin{array}{c}\text { Fabricación de } \\
\text { insumos textiles y } \\
\text { acabado de textiles }\end{array}$ & 313 & $\begin{array}{l}\text { UE dedicadas a la preparación e hilado de fibras } \\
\text { textiles naturales, a la fabricación de hilos y telas, y } \\
\text { al acabado y recubrimiento de textiles }\end{array}$ \\
\hline $\begin{array}{l}\text { Fabricación de } \\
\text { productos textiles, } \\
\text { excepto prendas de } \\
\text { vestir }\end{array}$ & 314 & $\begin{array}{l}\text { UE dedicadas a la fabricación de alfombras, tapetes } \\
\text { y esteras a partir de hilo comprado, a la confección } \\
\text { (corte y cosido) de cortinas, blancos y similares a } \\
\text { partir de tela comprada, y de otros productos } \\
\text { textiles }\end{array}$ \\
\hline $\begin{array}{l}\text { Fabricación de } \\
\text { prendas de vestir }\end{array}$ & 315 & $\begin{array}{l}\text { UE dedicadas a la fabricación de prendas de vestir } \\
\text { de tejido de punto, y a la confección de prendas de } \\
\text { vestir y accesorios de vestir }\end{array}$ \\
\hline $\begin{array}{l}\text { Curtido y acabado de } \\
\text { cuero y piel, y } \\
\text { fabricación de } \\
\text { productos de cuero, } \\
\text { piel, y materiales } \\
\text { sucedáneos }\end{array}$ & 316 & $\begin{array}{l}\text { UE dedicadas al curtido y acabado de cuero y piel, a } \\
\text { la fabricación de calzado y de productos de cuero, } \\
\text { piel y materiales sucedáneos, como bolsos de mano, } \\
\text { maletas y similares, y otros productos de cuero y } \\
\text { piel }\end{array}$ \\
\hline $\begin{array}{c}\text { Industria de la } \\
\text { madera }\end{array}$ & 321 & $\begin{array}{l}\text { UE dedicadas a la fabricación de diversos productos } \\
\text { de madera en aserraderos integrados, al aserrado } \\
\text { (corte) de tablas y tablones, a partir de la madera } \\
\text { en rollo, a la impregnación y tratamiento de } \\
\text { maderas }\end{array}$ \\
\hline Industria del papel & 322 & $\begin{array}{l}\text { UE dedicadas a la fabricación de pulpa (de madera y } \\
\text { de materiales reciclados), papel, cartón, y productos } \\
\text { de papel y cartón }\end{array}$ \\
\hline $\begin{array}{l}\text { Impresión de } \\
\text { industrias conexas }\end{array}$ & 323 & $\begin{array}{l}\text { UE dedicadas a la impresión y otros impresos, a la } \\
\text { impresión de formas continuas y a realizar } \\
\text { actividades para la industria de la impresión, como } \\
\text { la encuadernación y elaboración de placas, clichés, } \\
\text { grabados y otros productos similares }\end{array}$ \\
\hline $\begin{array}{l}\text { Fabricación de } \\
\text { productos derivados } \\
\text { del petróleo y del } \\
\text { carbón }\end{array}$ & 324 & $\begin{array}{l}\text { UE dedicadas a la refinación de petróleo crudo, a la } \\
\text { fabricación de productos de asfalto, aceites y grasas } \\
\text { lubricantes, y de otros productos derivados del } \\
\text { petróleo refinado y del carbón mineral }\end{array}$ \\
\hline Industria química & 325 & $\begin{array}{l}\text { UE dedicadas a la fabricación de productos químicos } \\
\text { básicos; de resinas y hules sintéticos; fibras } \\
\text { químicas, fertilizantes, pesticidas y otros } \\
\text { agroquímicos; productos farmacéuticos; pinturas, } \\
\text { recubrimientos y adhesivos; jabones, limpiadores y } \\
\text { preparaciones de tocador; tintas para impresión; } \\
\text { explosivos, y otros productos químicos }\end{array}$ \\
\hline $\begin{array}{l}\text { Industria del plástico } \\
\text { y del hule }\end{array}$ & 326 & $\begin{array}{l}\text { UE dedicadas a la fabricación de productos de } \\
\text { plástico y de hule }\end{array}$ \\
\hline Fabricación de & 327 & UE dedicadas a la fabricación de productos a base de \\
\hline
\end{tabular}


Tecnología y

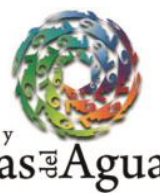

\begin{tabular}{|c|c|c|}
\hline $\begin{array}{l}\text { productos a base de } \\
\text { minerales no } \\
\text { metálicos }\end{array}$ & & $\begin{array}{l}\text { arcillas y minerales refractarios, de vidrio y } \\
\text { productos de vidrio; de cemento y productos de } \\
\text { concreto; de cal, yeso y productos de yeso; y de } \\
\text { otros productos a base de minerales no metálicos }\end{array}$ \\
\hline $\begin{array}{c}\text { Industrias metálicas } \\
\text { básicas }\end{array}$ & 331 & $\begin{array}{l}\text { UE dedicadas a la fundición primaria de hierro bruto; } \\
\text { a la fabricación de acero y productos de hierro y } \\
\text { acero; a la fundición, afinación, refinación y } \\
\text { laminación de metales no ferrosos, y al moldeo por } \\
\text { fundición de piezas metálicas }\end{array}$ \\
\hline $\begin{array}{l}\text { Fabricación de } \\
\text { productos metálicos }\end{array}$ & 332 & $\begin{array}{l}\text { UE dedicadas a la fabricación de productos forjados } \\
\text { y troquelados a partir de metal comprado; } \\
\text { herramientas de mano metálicas sin motor y } \\
\text { utensilios de cocina metálicos; partes y estructuras } \\
\text { metálicas y acero para la construcción y productos } \\
\text { de herrería, calderas industriales, tanques y envases } \\
\text { metálicos, herrajes y cerraduras; alambre, } \\
\text { productos de alambre y resortes al maquinado } \\
\text { hecho sobre pedido de piezas metálicas nuevas y } \\
\text { usadas para maquinaria y equipo en general; a la } \\
\text { fabricación de tornillos, tuercas, remaches y } \\
\text { similares; recubrimiento de piezas metálicas, y otros } \\
\text { terminados metálicos }\end{array}$ \\
\hline $\begin{array}{l}\text { Fabricación de } \\
\text { maquinaria y equipo }\end{array}$ & 333 & $\begin{array}{l}\text { UE dedicadas a la fabricación de maquinaria y } \\
\text { equipo para las actividades agropecuarias; } \\
\text { construcción, industria extractiva; para las industrias } \\
\text { manufactureras, el comercio y los servicios; de } \\
\text { equipo de aire acondicionado, calefacción, y de } \\
\text { refrigeración industrial y comercial; de motores de } \\
\text { combustión interna, turbinas y transmisiones; y de } \\
\text { otra maquinaria y equipo para la industria en } \\
\text { general }\end{array}$ \\
\hline $\begin{array}{l}\text { Fabricación de equipo } \\
\text { de computación, } \\
\text { comunicación, } \\
\text { medición y de otros } \\
\text { equipos componentes, } \\
\text { y accesorios } \\
\text { electrónicos }\end{array}$ & 334 & $\begin{array}{l}\text { UE dedicadas a la fabricación de computadoras y } \\
\text { equipo periférico; equipo de comunicación; equipo } \\
\text { de audio y video; componentes electrónicos; } \\
\text { instrumentos de medición, control, navegación; } \\
\text { equipo médico electrónico; y a la fabricación, y } \\
\text { reproducción masiva de medios magnéticos y } \\
\text { ópticos }\end{array}$ \\
\hline $\begin{array}{l}\text { Fabricación de } \\
\text { accesorios para } \\
\text { aparatos eléctricos y } \\
\text { equipo de generación } \\
\text { de energía eléctrica }\end{array}$ & 335 & $\begin{array}{l}\text { UE dedicadas a la fabricación de accesorios de } \\
\text { iluminación, aparatos eléctricos de uso doméstico, } \\
\text { equipo de generación y distribución de energía } \\
\text { eléctrica, y otros equipos y accesorios eléctricos }\end{array}$ \\
\hline $\begin{array}{c}\text { Fabricación de equipo } \\
\text { de transporte }\end{array}$ & 336 & $\begin{array}{l}\text { UE dedicadas a la fabricación de equipo de } \\
\text { transporte, como automóviles, camionetas y } \\
\text { camiones, carrocerías y remolques; partes para }\end{array}$ \\
\hline
\end{tabular}




\begin{tabular}{|c|c|l|}
\hline & & $\begin{array}{l}\text { vehículos automotores; equipo aeroespacial; equipo } \\
\text { ferroviario, para embarcaciones y otros equipos de } \\
\text { transporte }\end{array}$ \\
\hline $\begin{array}{c}\text { Fabricación de } \\
\text { muebles, colchones y } \\
\text { persianas }\end{array}$ & 337 & $\begin{array}{l}\text { UE dedicadas a la fabricación de muebles, colchones, } \\
\text { persianas y cortineros, incluye fabricación de } \\
\text { restiradores y pizarrones, de colchones de espuma } \\
\text { de uretano y colchones de agua }\end{array}$ \\
\hline Otras industrias & 339 & $\begin{array}{l}\text { UE dedicadas a la fabricación de equipo y aparatos } \\
\text { no electrónicos para uso médico, dental y para } \\
\text { laboratorio; material desechable de uso médico y de } \\
\text { artículos ópticos de uso oftalmológico, y otras } \\
\text { manufacturas no clasificadas en otra parte }\end{array}$ \\
\hline
\end{tabular}

\section{Resultados}

\section{Estimación de elasticidades $(\varepsilon)$ y valor marginal del agua $(\rho)$}

Los resultados de la estimación de los modelos para toda la industria manufacturera de la Subregión Hidrológica Río San Juan (SHRSJ) se presentan en la Tabla 3 . Un $80 \%$ de las variables de la función CobbDouglas (CD) es estadísticamente significativa desde un punto de vista individual; mientras que este valor es del $60 \%$ para la función TransLogarítmica (TL) en ambos modelos, en su gran mayoría de variables a un $99 \%$ de significancia. El ajuste estadístico $\left(R^{2}\right)$ en la función CD es del $90 \%$, lo cual indica que $90 \%$ del total de la variación en la variable dependiente se explica por el conjunto de las variables independientes. En el caso de la función TL, dicho ajuste es igual a un $91 \%$. Además, el valor de la prueba Fisher (Prob > F) en ambos modelos rechaza la hipótesis nula de que todos los coeficientes de manera simultánea son iguales a cero (Tabla 3 ). 
Tecnología y

Ciencias $\stackrel{\unlhd}{\unlhd}$ gua

Tabla 3. Resultados de la estimación de los modelos econométricos. Fuente: elaboración propia con base en el Censo Económico 2014 del Instituto Nacional de Estadística y

Geografía (INEGI, 2017).

\begin{tabular}{|c|c|c|c|c|c|c|}
\hline \multirow{3}{*}{$\begin{array}{l}\text { Variable } \\
\ln L\end{array}$} & \multicolumn{3}{|c|}{ Función Cobb-Douglas (CD) } & \multicolumn{3}{|c|}{ Función Trans-Log (TL) } \\
\hline & \multirow{2}{*}{$\begin{array}{c}\text { Estimado } \\
0.012\end{array}$} & \multicolumn{2}{|c|}{ Significancia } & \multirow{2}{*}{$\begin{array}{c}\text { Estimado } \\
-0.446\end{array}$} & \multicolumn{2}{|c|}{ Significancia } \\
\hline & & 0.614 & & & 0.023 & $*$ \\
\hline $\ln W$ & 0.037 & 0.007 & $* * *$ & 0.166 & 0.185 & \\
\hline $\ln M$ & 0.862 & 0.000 & $* * *$ & 0.872 & 0.000 & $* * *$ \\
\hline $\ln K$ & 0.072 & 0.000 & $* * *$ & 0.172 & 0.321 & \\
\hline $\ln L * \ln W$ & & & & -0.011 & 0.570 & \\
\hline $\ln L * \ln M$ & & & & 0.092 & 0.000 & $* * *$ \\
\hline $\ln L * \ln K$ & & & & -0.023 & 0.276 & \\
\hline $\ln W * \ln M$ & & & & -0.044 & 0.000 & $* * *$ \\
\hline $\ln W * \ln K$ & & & & 0.039 & 0.003 & $* * *$ \\
\hline $\ln M * \ln K$ & & & & -0.040 & 0.004 & $* * *$ \\
\hline$(\ln L)^{2}$ & & & & -0.055 & 0.004 & $* * *$ \\
\hline$(\ln W)^{2}$ & & & & 0.001 & 0.869 & \\
\hline$(\ln M)^{2}$ & & & & 0.017 & 0.003 & $* * *$ \\
\hline$(\ln K)^{2}$ & & & & 0.009 & 0.366 & \\
\hline$A$ & 1.425 & 0.000 & $* * *$ & 1.195 & 0.214 & \\
\hline$N^{\circ}$ Obs. & & & & & 54 & \\
\hline Prob $>F$ & & & & & 00 & \\
\hline$R^{2}$ & & & & & 91 & \\
\hline
\end{tabular}

Significancia estadística: $*=10 \%, * *=5 \%$ y $* * *=1 \%$

La forma funcional $C D$ registra una elasticidad del agua $\left(\varepsilon_{C D-w}\right)$ en toda la industria manufacturera igual a 0.037 , y el valor marginal del agua $\left(\rho_{C D-w}\right)$ un valor de USD 10.5 por cada $\mathrm{m}^{3}$ consumido y adicionado al proceso productivo.

En la función $T L$, la elasticidad del producto con respecto al consumo de agua para toda la industria manufacturera $\left(\varepsilon_{T L-w}\right)$ es igual a 0.046 , y el $\rho_{T L-W}$ es igual a USD 13.0 por cada $\mathrm{m}^{3}$, resultado un poco superior a lo encontrado a través de la forma funcional CD (Tabla 3). Sin embargo, y como ya se mencionó, la literatura expresa preferencia por la forma función TL a la forma función CD debido a que esta última es demasiado 


\section{Ciencias $₫$ Agua}

restrictiva en comparación con la función $T L$, que es una forma más general de la función de producción (Tabla 4).

Tabla 4. Elasticidad $\left(\varepsilon_{W}\right)$ y valor marginal del producto con respecto al uso de agua ( $\left.\rho_{w}\right)$ en el sector manufacturero Fuente: elaboración propia con base en el Censo Económico 2014 del Instituto Nacional de Estadística y Geografía (INEGI, 2017).

\begin{tabular}{|c|c|c|c|c|c|}
\hline \multirow{2}{*}{ Sectores } & \multirow{2}{*}{ Código } & \multicolumn{2}{|c|}{$\begin{array}{l}\text { Función Cobb- } \\
\text { Douglas (CD) }\end{array}$} & \multicolumn{2}{|c|}{$\begin{array}{c}\text { Función Translog } \\
\text { (TL) }\end{array}$} \\
\hline & & $\varepsilon_{C D-w(\%)}$ & $\begin{array}{r}\rho_{C D-w} \\
\left(U S D / m^{3}\right)\end{array}$ & $\varepsilon_{T L-W(\%)}$ & $\begin{array}{r}\rho_{T L-w_{3}} \\
\left(U S D / m^{3}\right)\end{array}$ \\
\hline $\begin{array}{l}\text { Todas las industrias } \\
\text { manufactureras }\end{array}$ & $\begin{array}{c}31,32 y \\
33\end{array}$ & 0.037 & 10.5 & 0.046 & 13.0 \\
\hline Alimentos & 311 & 0.027 & 9.7 & 0.024 & 9.1 \\
\hline Bebidas y tabaco & 312 & 0.071 & 14.1 & 0.068 & 13.1 \\
\hline $\begin{array}{l}\text { Fabricación de prendas de } \\
\text { vestir }\end{array}$ & 315 & 0.029 & 3.9 & 0.026 & 3.7 \\
\hline $\begin{array}{l}\text { Curtido y acabado de cuero } \\
\text { y piel }\end{array}$ & 316 & 0.056 & 18.9 & 0.038 & 12.8 \\
\hline Papel & 322 & 0.061 & 14.4 & 0.053 & 12.8 \\
\hline Química & 325 & 0.073 & 8.8 & 0.099 & 13.4 \\
\hline \begin{tabular}{|l|} 
Productos a base de \\
minerales no metálicos
\end{tabular} & 327 & 0.021 & 7.3 & 0.028 & 10.1 \\
\hline $\begin{array}{l}\text { Fabricación de productos } \\
\text { metálicos }\end{array}$ & 332 & 0.009 & 1.3 & 0.053 & 7.3 \\
\hline $\begin{array}{l}\text { Fabricación de maquinaria y } \\
\text { equipo }\end{array}$ & 333 & 0.024 & 14.2 & 0.066 & 11.2 \\
\hline $\begin{array}{l}\text { Equipo de computación, } \\
\text { comunicación y medios }\end{array}$ & 334 & 0.024 & 0.4 & 0.047 & 0.9 \\
\hline Equipo de transporte & 336 & 0.082 & 15.9 & 0.075 & 15.4 \\
\hline $\begin{array}{l}\text { Fabricación de muebles, } \\
\text { colchones y persianas }\end{array}$ & 337 & 0.013 & 5.8 & 0.028 & 9.6 \\
\hline $\begin{array}{l}\text { Otras industrias } \\
\text { manufactureras }\end{array}$ & 339 & 0.083 & 11.1 & 0.073 & 8.5 \\
\hline
\end{tabular}

De igual forma, la Tabla 4 presenta los resultados para los diferentes sectores que conforman la industria manufacturera de la SHRSJ tanto para una forma funcional CD como TL. La estimación de la elasticidad del producto con respecto al consumo de agua presenta un rango que 
va desde 0.024 para el sector de alimentos hasta un valor igual a 0.099 para el sector de productos químicos.

Por otro lado, el valor marginal del agua presenta un rango que va desde USD 0.9 por cada $\mathrm{m}^{3}$ para el caso del sector de equipo de computación, comunicación y persianas, hasta USD 15.4 para el caso del sector de equipo de transporte.

Si el valor marginal del agua $\left(\rho_{w}\right)$ se compara con la participación en el valor total de la producción y con el uso total del agua usado en el proceso productivo, se observa que los sectores más eficientes son transporte, productos químicos y alimentos (Figura 2).

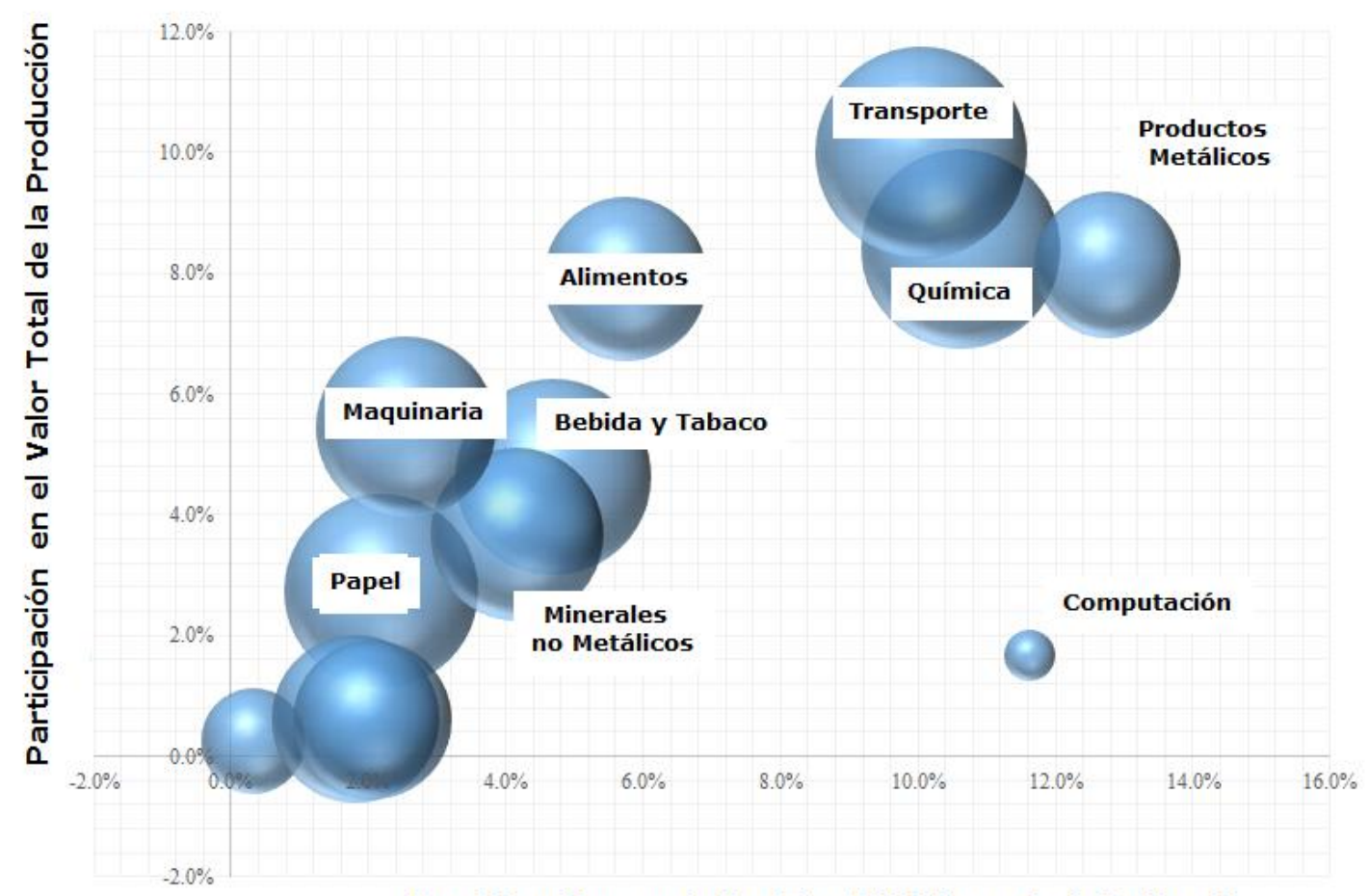

Participación en el Uso Total del Agua de la Industria

Figura 2. Valor marginal del producto $\left(\rho_{w}\right)$ con respecto a la participación total del valor de la producción y uso total del agua en la industria manufacturera. Fuente: elaboración propia, 2017. 


\section{Conclusiones y recomendaciones}

A pesar de la importancia del uso industrial del agua y de los aspectos que lo distinguen de otros usos, la función del agua en la industria permanece poco estudiada. Esto es más cierto para los países en vías de desarrollo o emergentes (Renzetti, 2002). Para el caso de la Subregión Hidrológica Río San Juan (SHRSJ), los resultados encontrados para modelar el comportamiento del uso del agua en la industria manufacturera confirman que existe sensibilidad a variables económicas. En específico, se estimó que el valor marginal del agua es igual a USD 13.0 por cada $\mathrm{m}^{3}$ usado en el proceso de producción. A nivel internacional, las estimaciones del mismo indicador oscilan entre USD $0.01 / \mathrm{m}^{3}$ a USD $7.00 / \mathrm{m}^{3}$, para países de Asia y Norteamérica (excluyendo México) (Aylward et al., 2010), mientras que el caso de Chile se observa un valor promedio para la industria manufacturera de USD $30.9 / \mathrm{m}^{3}$ (Vargas, 2015).

En ese sentido, el valor encontrado en esta investigación, si bien está por encima de las estimaciones promedio a nivel internacional, se puede explicar principalmente por el bajo costo al que tienen acceso al recurso agua las unidades económicas (UE) del país, y en este caso de la zona de estudio, que está por debajo de los precios a los cuales la adquieren las UE en otros países.

Los costos que deben desembolsar las UE en el país - no sólo del sector manufacturero sino de toda la industria- se establecen en la "Ley Federal de Derechos - Disposiciones Aplicables en Materia de Aguas Nacionales 2016". Dichos costos dependen de la Zona de Disponibilidad del recurso natural, y si son aguas provenientes de fuentes superficiales o extraídas del subsuelo.

En el caso de la Zona de Disponibilidad y de agua subterráneas se clasifican en cuatro zonas, tomando en cuenta el Índice de Disponibilidad (Idas), que se calcula como Idas = Dma / $(R-D n c)$, donde $D m a$ es la disponibilidad media anual de agua subterránea en una unidad hidrogeológica; $R$, la recarga total media anual, y Dnc es la descarga natural comprometida.

Así, se establece que la Zona de Disponibilidad 1 presenta un Idas menor o igual a -0.1 , y se debe cubrir USD $1.12 / \mathrm{m}^{3}$ que se utilice de 
esa zona. En el caso de la Zona de Disponibilidad 2, el Idas es mayor a 0.1 y menor o igual a 0.1 , y debe cubrir USD $0.44 / \mathrm{m}^{3}$. En la Zona de Disponibilidad 3, el Idas es mayor a 0.1 y menor o igual a 0.8 , y se debe cubrir USD $0.14 / \mathrm{m}^{3}$. Al final, en la Zona de Disponibilidad 4, el Idas es mayor a 0.8 y se deben cubrir USD $0.10 / \mathrm{m}^{3}$.

En el caso de la SHRSJ, que pertenece a la Zona 1, las UE desembolsan en promedio USD $1.12 / \mathrm{m}^{3}$; mientras que, en promedio, en los países de la Organización para la Cooperación y el Desarrollo (OCDE) es de USD $1.38 / \mathrm{m}^{3}$ (Walsh, Cusack, \& O'Sullivan, 2016). Es decir, un valor inferior del orden de $21 \%$ aproximadamente.

La industria manufacturera utiliza el agua como un insumo para una variedad de productos; por lo tanto, dicho recurso es uno de los recursos más importantes en el proceso de producción. Además, una fuente estable de agua es esencial para las empresas manufactureras, porque ayuda a evitar problemas derivados de la escasez y para generar valor añadido a su producto. Sin embargo, pocas investigaciones han analizado el agua de esta industria y su valor añadido en países en desarrollo o emergentes, como es el caso de México, o como es en este caso en la SHRSJ.

En México, el precio del agua por lo general es regulado por el gobierno del estado, por lo que la importancia de éste y su valor añadido en los bienes finales no se ha considerado en su real dimensión.

Esta investigación estimó la elasticidad del producto $\left(\varepsilon_{W}\right)$ y el valor marginal del agua $\left(\rho_{w}\right)$ para el sector industrial manufacturero que se ubica en la Subregión Hidrológica Río San Juan (SHRSJ), usando información de casi 18 mil unidades económicas (UE), agrupadas a nivel municipal a través de la estimación de modelos econométricos, considerando formas funcionales de producción tipo Cobb-Douglas (CD) y Trans-Logarítmica (TL).

La elasticidad del producto con respecto al agua para toda la industria manufacturera y el valor marginal del agua en la SHRSJ se estimaron en 0.046 y USD 13.0 por cada $\mathrm{m}^{3}$ usado en el proceso de producción, respectivamente. El $\rho_{w}$ estimado osciló entre USD 0.9 por cada $\mathrm{m}^{3}$ para el sector de equipo de cómputo hasta USD 15.4 para el sector de equipo de transporte, reflejando que éste varía según el sector analizado.

Desde el punto de vista de política pública, la información encontrada en este tipo de investigación puede servir para identificar y cuantificar el valor en términos monetarios de los impactos en inversiones públicas 
destinadas a ofrecer el recurso agua, ya sea al sector manufacturero o a algún subsector que pertenece a éste. Es decir, se puede calcular el beneficio monetario del suministro de agua a una industria como resultado de un nuevo proyecto de dotación del recurso. Por ejemplo, en el caso de la Subregión Hidrológica Río San Juan (SHRSJ), en promedio cada nuevo $\mathrm{m}^{3}$ de agua que se destine al sector de la industria manufacturera procedente de una nueva inversión en la dotación del recurso lograría un beneficio económico adicional de unos USD 13.0 en el proceso productivo. Lo importante es que el costo por cada $\mathrm{m}^{3}$ de agua en que incurra el estado o la empresa prestadora del servicio de agua tenga un valor menor que dicho monto, con la idea que dicha inversión sea viable y rentable.

Asimismo, las UE que se ubican en la SHRSJ gastan en promedio USD 1.01 por cada $\mathrm{m}^{3}$ que ingresa al proceso de producción, por lo cual el gobierno del estado tiene cierto margen para considerar ajustes en las tarifas que cobra por el uso de agua para procesos de producción, en especial en cuencas que presentan problemas de escasez del recurso natural. Este resultado permite reflexionar sobre el margen de maniobra que tiene el estado para aumentar los precios por suministro de agua en la industria, incluyendo los costos que implican los daños a los acuíferos sobreexplotados. El impacto de esta modificación de precios serviría para mejorar la eficiencia en su uso y en la ubicación de los recursos, aumentar los ingresos del estado y mejorar la base de conocimiento gubernamental con respecto al uso del agua.

Por otro lado, en situaciones de estrés del recurso hídrico, como se evidencia en la SHRSJ, se debería considerar la eficiencia en el uso que se le dé a éste; por ejemplo, desde el punto de vista del valor agregado que puede ofrecer cada subsector del sector manufacturero en la producción por cada $\mathrm{m}^{3}$ usado en el proceso. Así, un $\mathrm{m}^{3}$ destinado en el sector de alimentos genera unos USD $9.1 / \mathrm{m}^{3}$ como valor agregado; mientras que el mismo $\mathrm{m}^{3}$ destinado al sector de productos químicos genera USD 13.4; es decir, 1.5 veces más de valor. Sin embargo, es necesario indicar que este valor agregado es enteramente desde un punto de vista económico y no considera los efectos a terceros, como pueden ser las externalidades negativas que pueden ser diversas y de distinta magnitud, dependiendo del subsector analizado y de los agentes económicos involucrados. En ese sentido, si bien el uso de los valores hallados en la estimación del valor marginal del agua entre subsectores puede ser una herramienta útil para la toma de decisiones para reasignar usos del agua, sobre todo cuando se trata de problemas de 
escasez de agua, es necesario complementar con estudios sobre valoraciones socioeconómicas de impacto sobre bienes y servicios ambientales.

La información obtenida en esta investigación proporciona criterios técnicos razonables para la toma de decisiones sobre la asignación y uso del agua de manera eficiente. Por ejemplo, en cuencas hídricas con problemas de escasez de agua, este tipo de análisis podría servir para determinar qué subsectores -ya sean dentro de la misma industria manufacturera o dentro de otros sectores (hogares, agricultura, entro otros) - deberían tener cierto grado de preferencia al acceso al agua, acompañando estos estudios con consideraciones sociales y ambientales.

De igual forma, este tipo de hallazgos debería concientizar a la sociedad, a las autoridades y principalmente a los usuarios de este recurso natural, tomando en cuenta su valor dentro de un ambiente de escasez y la necesidad de considerar al agua recurso vital en todos los procesos productivos.

Por tanto, se espera que las estimaciones de este tipo de estudios sean útiles para el diseño de futuras políticas de tarifas y manejo del agua por parte de los gobiernos o administraciones encargadas de este recurso natural escaso.

Finalmente, es necesario avanzar en el conocimiento del uso de agua industrial y generar instrumentos que permitan lograr un manejo racional del agua, para así obtener mejores y mayores beneficios para la sociedad.

Como recomendación para futuros estudios, se podrían considerar variables ambientales y sociales, como precipitación; recarga de acuíferos de donde extraen el agua las empresas; grado de explotación de los diferentes acuíferos; rigidez en los mercados de los insumos, y huelgas laborales, entre otros, en la especificación de las formas funciones de la producción (Cobb-Douglas y/o Translogarítmica). Si bien estas variables no se consideran como un insumo directo dentro del proceso productivo, podrían ser variables exógenas al proceso, que podrían tener cierto grado de impacto.

Por otro lado, los resultados hallados en este tipo de trabajo podrían servir para medir los impactos económicos a terceros, que se ocasionan en el proceso de producción; en economía, las famosas externalidades negativas. 


\section{Agradecimientos}

El artículo se elaboró con el apoyo financiero del Consejo Nacional de Ciencia y Tecnología (Conacyt), en el marco del proyecto "Hacia una Gestión Integral del Agua por Cuenca Hidrográfica: un Análisis de Disponibilidad y Usos, Fondo Problemas Nacionales" (proyecto Núm. 120-2015), y con el respaldo del Programa de Cátedras Conacyt.

\section{Referencias}

Aguilar-Benitez, I. (2017). Uso urbano del agua en el contexto de sus subregiones hidrológicas: Bajo Grijalva, Valle de México y San Juan. Cuencas de México, 8, 3-9.

Aylward, B., Seely, H., Hartwell, R., \& Dengel, J. (2010). The economic value of water for agricultural, domestic and industrial uses: $A$ global compilation of economic studies and market prices. Rome, Italy: Food and Agriculture Organization of the United Nations (FAO).

Baumann, D. D., Boland, J. J., \& Hanemann, W. M. (1997). Urban Water Demand Management and Planning. USA: McGraw-Hill, Inc.

Bruneau, J., Renzetti, S. J., \& Villeneuve, M. (2010). Manufacturing firms' demand for water recirculation. Canadian Journal of Agricultural Economics, 58(4), 515-530.

Christensen, L., Jorgenson, D., \& Lau, L. (1973). Transcendental logarithmic production function frontiers. The Review of Economics and Statistics, 55, 29-45.

Conagua, Comisión Nacional del Agua. (2012a). Programa Hídrico Regional, Región Hidrológico-Administrativa XIII Aguas del Valle de México. México, DF, México: Comisión Nacional del Agua.

Conagua, Comisión Nacional del Agua (2012b). Programa Hídrico Regional, Región Hidrológico-Administrativa VI Río Bravo, México. México, DF, México: Comisión Nacional del Agua. 
Conagua, Comisión Nacional del Agua. (2016). Estadísticas del agua en México. Ciudad de México, México: Comisión Nacional del Agua.

De-Gispert, C. (2004). The economic analysis of industrial water demand: A review. Environment and Planning C: Politics and Space, 22(1), 15-30.

De-Rooy, J. (1974). Price responsiveness of the industrial demand for water. Water Resources Research, 10(3), 403-406.

Dupont, D. P., \& Renzetti, S. J. (2001). The role of water in manufacturing. Environmental and Resource Economics, 18(4), 411-432.

Féres, J., Reynaud, A., \& Thomas, A. (2012). Water reuse in Brazilian manufacturing firms. Applied Economics, 44(11), 1417-1427.

Féres, J., \& Reynaud, A. (2003). Industrial water use, cost structure and environmental policy in Brazil. Recuperado de http://www2.toulouse.inra.fr/lerna/cahiers2003/0308114.pdf

Grebenstein, C. R., \& Field, B. C. (1979). Substituting for water inputs in U.S. manufacturing. Water Resources Research, 15(2), 228-232.

Hussaina, I., Thrikawalaa, S., \& Barkera, R. (2002). Economic analysis of residential, commercial, and industrial uses of water in Sri Lanka. Water International, 27(2), 183-193.

$\mathrm{Ku}$, S. J., \& Yoo, S. H. (2012). Economic value of water in the Korean manufacturing industry. Water Resource Management, 26, 81-88. Recuperado de https://link.springer.com/article/10.1007/s11269011-9905-z

Malla, P. B., \& Gopalakrishnan, C. (1999). The economics of urban water demand: The case of industrial and commercial water use in Hawaii. Water Resources Development, 15(3), 367-374.

Marcouiller, D., \& Coggins, S. (1999). The economic value of water: An introduction. Water Issues in Wisconsin, G3698. Recuperado de https://fred.ifas.ufl.edu/pdf/conservation-webinars/WisconsinG3698_1.pdf

Oh, H. S. (1973). Economics of urban water demand: A case study of the Honolulu board of water supply (tesis de doctorado). University of Hawaii in Manoa, Hawaii.

Onjala, J. (2001). Industrial water demand in Kenya: Industry behavior when tariffs are not binding (tesis de doctorado). Roskilde 
University Centre, Department of Environment, Roskilde, Denmark.

Rees, J. A. (1969). Industrial demand of water: A study of South East England. London, United Kingdom: Weidenfeld \& Nicolson.

Renzetti, S. J. (2002). The economics of industrial water use. Cheltenham, UK, Northampton, MA, USA: Edward Elgar Pub. Recuperado de https://www.amazon.com/Economics-IndustrialWater-Management-Resources/dp/1840644990

Renzetti, S. J., \& Dupont, D. P. (2002). The value of water in manufacturing. Working Paper ECM 03-03. Swindon, United Kingdom: Economic and Social Research Council, Centre for Social Economic Research on the Global Environment.

Reynaud, A. (2003). An econometric estimation of industrial water demand in France. Environmental and Resource Economics, 25(2), 213-232.

Vargas, L. J. (2015). Valor económico del agua en la industria manufacturera chilena: enfoque de productividad marginal de factores (tesis de maestría). Universidad de Concepción, Facultad de Ciencias Económicas y Administrativas, Concepción, Chile.

Walsh, B. P., Cusack, D. O., \& O'Sullivan, D. T. J. (2016). An industrial water management value system framework development. Sustainable Production and Consumption, 5, 82-93.

Wang, H., \& Lall, S. (2002). Valuing water for Chinese industries: A marginal productivity analysis. Applied Economics, 34(6), 759765.

William, S. (1986). The demand for water by customer class. Applied Economics, 18(12), 1275-1289. 